\title{
Physical Strain and Risk during Stroller Locomotion on Cross and Combined Slopes
}

\author{
Satoshi Muraki ${ }^{1, a}$, Seiji Saito ${ }^{2}$ and Mituru Ookura ${ }^{3}$ \\ ${ }^{1}$ Faculty of Design, Kyushu University, 4-9-1 Shiobaru Minami-ku Fukuoka, 815-8540, Japan \\ ${ }^{2}$ Matsue College of Technology, 14-4 Nishiikuma-cho, Matsue, Shimane, 690-8518, Japan \\ ${ }^{3}$ Faculty of Nursing, Japanese Red Cross Kyushu International College of Nursing, 1-1 Asty \\ Munakata, Fukuoka, 811-4157, Japan \\ a muraki@design.kyushu-u.ac.jp
}

Keywords: sidewalk, cross slope, combined slope, stroller, electromyogram

\begin{abstract}
In sidewalks, slopes for vehicle crossings are often installed, and pedestrians have to walk across these slopes. The present study examined the influences of slope gradients on the danger and physical strain during stroller locomotion. Nine female participants walked forward while pushing a stroller with a dummy baby on a mock sidewalk with 1) a cross slope (the whole surface of the sidewalk tilted sideways) at gradients of $0 \%, 5 \%, 10 \%$, and $15 \%$, and 2) combined slopes connecting a cross slope at gradients of $0 \%, 5 \%, 10 \%$, and $15 \%$ and a flat $0 \%$ area. The velocity and tracks of the stroller, the pressure between the palm and the stroller handle, and an electromyogram (EMG) of the upper limbs of the participant were measured during stroller locomotion. Throughout both the cross and the combined slopes, the participants decreased their locomotion velocity according to the increase in the slope gradient. The steeper slope led the stroller to drift off-course to the downhill direction and caused increased pressure between the palm of the participant and the handle and increased EMG values, especially in the forearms. The results indicated that, on a sidewalk with a steeper cross slope, it is difficult for stroller users to maintain their intended course, even if they decrease their locomotion velocity and make their greater physical effort.
\end{abstract}

\section{Introduction}

In Japan, sidewalk surfaces are raised above the surface of a roadway to protect pedestrians and to prevent water pools on the sidewalk during and after precipitation. However, slopes in the sidewalk are often installed for vehicles to cross the sidewalk (Fig. 1a) to enter a parking area. These slopes form cross slopes perpendicular to the direction of the pedestrians. Furthermore, these slopes can be formed into combined slopes that connect a cross slope to a flat area. In busy town streets, many sidewalk slopes for vehicle crossings may be installed, and then the sidewalks take a wave-like shape (Fig. 1b). It is possible that these slopes cause pedestrians to suffer greater physical strain, especially when they are pushing wheeled aids such as a wheelchair or stroller. In addition, pushing a wheeled aid exposes pedestrians to greater danger and a possible loss of control on their intended course.

Some previous studies reported that physical strain due to cross and combined slopes is especially a problem for wheelchair, cane, crutch and brace users [1-4]. Stroller users can also suffer physical strain and risk by walking on cross and combined slopes. Strollers are the most popular tools to assist locomotion with a baby (or babies). Although previous studies examined the physical strain from a longitudinal slope (in the traveling direction of the pedestrian) during stroller locomotion [5], we know of no other studies that have focused on the cross and the combined slopes. Before the present study, we interviewed 60 stroller users and asked about inconvenient and hazardous areas outside the home [6]. In the case of sidewalks, some respondents pointed out that cross and combined slopes make it difficult for them to proceed on their intended course; in fact, the number of such responses was equal to or higher than responses noting the difficulty of steps and longitudinal slopes. Furthermore, most stroller users avoided steep cross and combined slopes and walked on the flat area or gentler slope on the far side of the road. Thus, the cross and combined slopes in sidewalks are possible hazards for stroller users. To protect the stroller user and a baby (babies) in the stroller, 
studies are necessary to examine the influences of sidewalk slope gradients on the physical strain and danger during stroller locomotion.

Therefore, the present study constructed an experimental mock sidewalk and examined the physical strain and danger during stroller locomotion by female adults. The variables included the locomotion behaviors of the stroller, the pressure between the palm of the user and the handle (hereafter, "PPH") to measure the gripping force, and electromyograms (EMGs) of the muscle activity in the upper limbs. In Japan, the "Act on Promotion of Smooth Transportation, etc. of Elderly Persons, Disabled Persons, etc." stipulates that the gradient of the cross slope should be less than 1\% [7]. In the USA, the Americans with Disability Act (ADA) Accessibility Guidelines for Buildings and Facilities (ADAAG) states requires a cross slope at or below 2\% [8]. However, our investigation revealed that most of the cross slopes in sidewalks in towns exceeded these standards. Furthermore, in many cases the slopes for vehicle crossing in some sidewalks remained after they were no longer needed. It is also difficult for a sidewalk of short width to fulfill that standard. Accordingly, the present study focused on cross slopes at the gradients of $0 \%, 5 \%, 10 \%$, and $15 \%$, and combined slopes (the connecting area between cross slopes and flat areas), all of which exist in today's sidewalks.

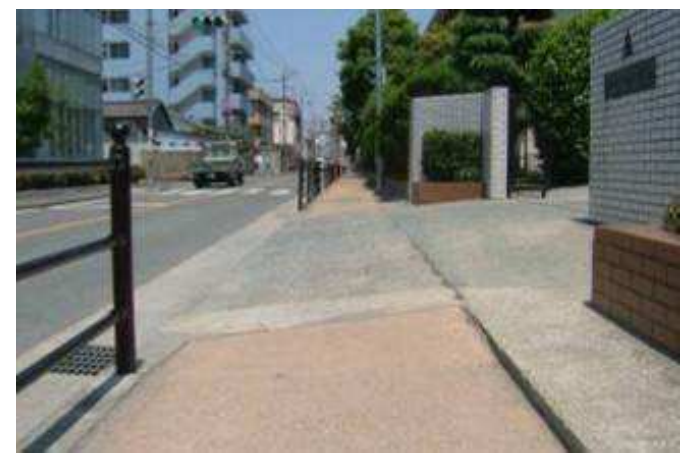

(a)

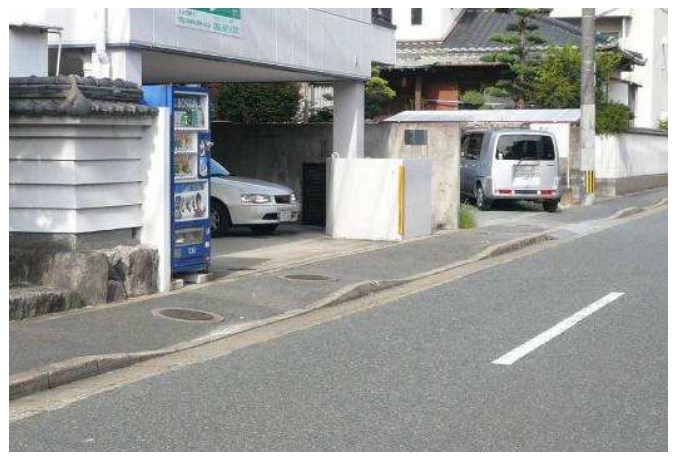

(b)

Fig. 1. Sidewalks with a cross slope. (a) The entire sidewalk tilts sideways. (b) Parts of the sidewalk tilt sideways to create a combined slope connecting a cross slope to a flat area. As shown, one cross slope (left side of (b)) is no longer needed.

\section{Methods}

Participants. Nine healthy adult females in their 20 s volunteered to participate in this study. The mean (range) of their ages and body heights were 22.8 (21 to 25) years and $157.1(150.0$ to 162.3$) \mathrm{cm}$, respectively. All participants (users) were right-handed and had little experience in using a stroller. Written informed consent was obtained from all participants before starting the study.

Mock sidewalk. A mock sidewalk (width $1.5 \mathrm{~m}$, length $6 \mathrm{~m}$ ) was constructed in a room. The sidewalk was made of wood, and rough, thick paper sheets were placed on the wood surface to imitate a real sidewalk. The sidewalk could be modified into three shapes: flat, cross slope, and combined slope. Under the flat condition $(0 \%)$, the entire surface of the sidewalk was flat. Under the cross slope condition, the entire surface of the sidewalk was tilted in the cross direction (perpendicular to the traveling direction of the pedestrians). The tilt could be changed to three gradients above $0 \%(5 \%$, $10 \%$, and $15 \%$ ). Under the combined slope condition, the sidewalk had one cross slope area (length $1200 \mathrm{~mm}$, Fig. 2 (a)) and two combined areas (length $900 \mathrm{~mm}$, both sides of cross slope area, Fig. 2 (b) and (c)), which imitated a real sidewalk with a vehicle crossing area (Fig. 3). The combined areas were transformed to connect between the cross slope and its flat side areas. The areas where the participants went down and up are defined as "Down" and "Up" (Fig. 2). The tilt of the area at the cross slope area (Fig 2 (a)) could be changed from $0 \%$ to gradients 5\%,10\%, and $15 \%$. The length of the area where the combined area was connected to its flat area was fixed to $900 \mathrm{~mm}$ based on our previous general survey in a town. 


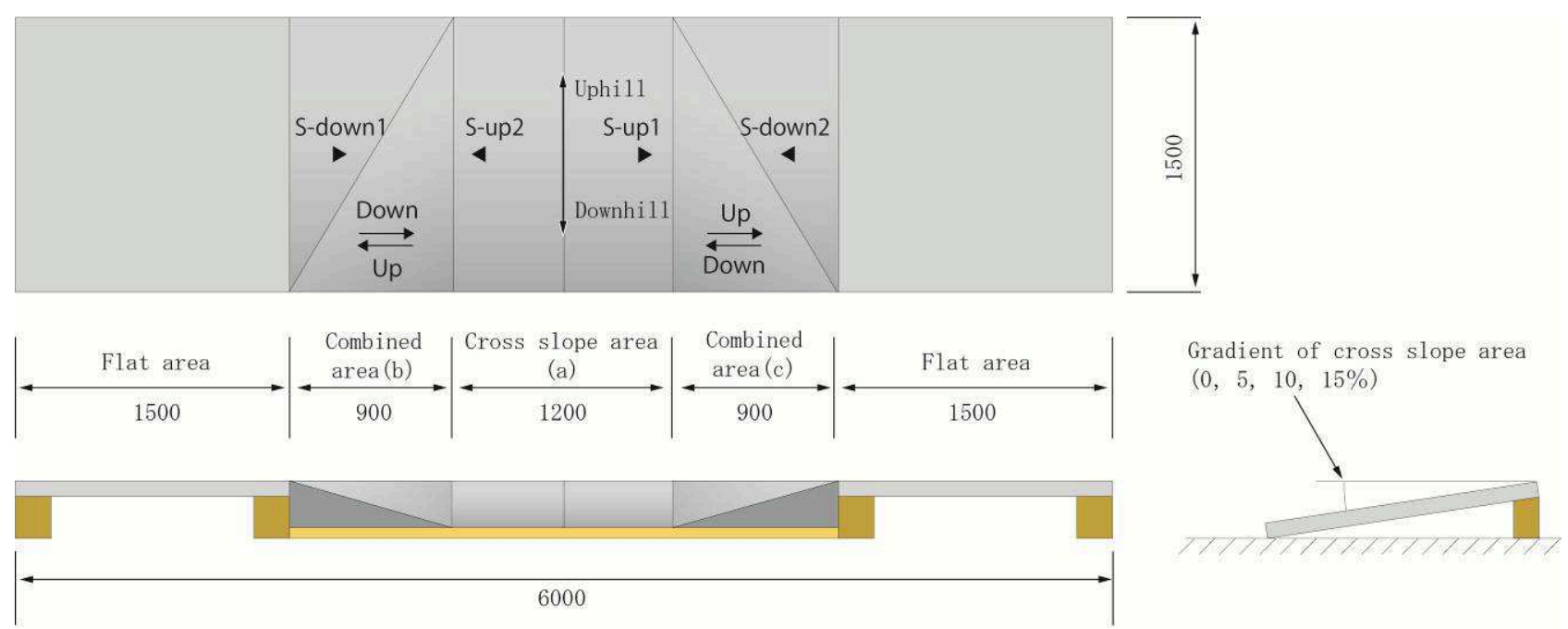

Fig. 2. Outline of the mock sidewalk under the combined slope condition. S-down and S-up are marks for the starting positions.

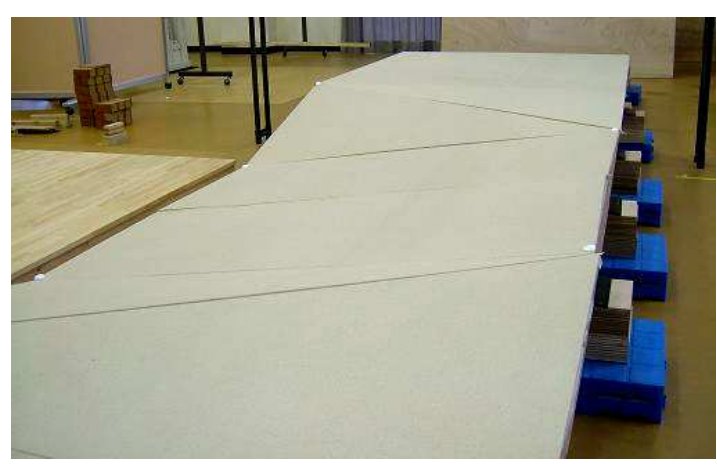

Fig. 3. Appearance of the mock sidewalk under the combined slope condition with a $15 \%$ gradient.

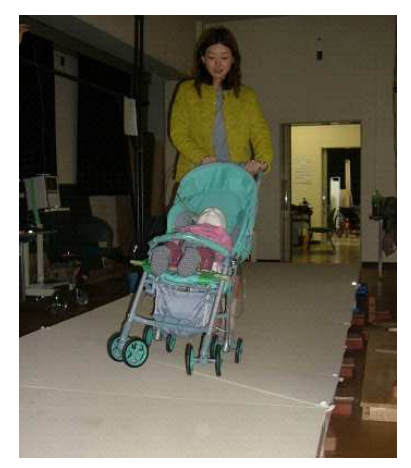

Fig. 4. Participant pushing a stroller with a child dummy on the mock sidewalk.

Procedures. This study used a folding stroller (Combi Co. Japan, Welflat ST-430, height $960 \mathrm{~mm}$, width $470 \mathrm{~mm}$, length $710 \mathrm{~mm}$ when opened), intended for children of ages 1-24 months. This stroller, shown in Fig. 4 with a participant, is one of the popular types in Japan. The dummy (height $75.3 \mathrm{~cm}$, weight $9.6 \mathrm{~kg}$ ), substituted for a child of age 12 months, was fastened by a seat belt equipped on the seat of the stroller. The height of the stroller handle was arranged according to each participant's height. Before participating in the experiment, the participants received instruction for the pushing method of the stroller and had practice time to become accustomed to pushing a stroller on the mock sidewalk with and without cross and combined slopes.

The participants executed stroller locomotion on the sidewalk under the following seven conditions: flat condition at one gradient $(0 \%)$, cross slope condition at three gradients $(5 \%, 10 \%$, and $15 \%)$, and combined slope condition at three gradients of the cross slope area $(5 \%, 10 \%$, and $15 \%)$. Furthermore, the combined slope condition had two types of stroller locomotion; going down and up the combined area (in other words, moving on the Down and Up areas, respectively). Under the cross slope and combined slope conditions, the participants pushed the stroller forward in two directions (right to left end sides, and left to right end sides).

The procedure for stroller locomotion is as follows. First, a participant stood on the sidewalk and held the handle of the stroller with both hands. Second, she moved the front casters of the stroller to the starting position. Under the cross slope condition, the starting positions were $1500 \mathrm{~mm}$ away from the end of the sidewalk. Under the combined slope condition, the starting positions for going down and up the combined areas were marked S-down (1 or 2) and S-up (1 or 2) in Fig. 2, respectively. Third, she pushed the stroller forward at a self-selected speed. Finally, under the cross slope condition, 
after moving forward for a given distance (minimum of $2 \mathrm{~m}$ ), she slowed down and stopped. Under the combined slope condition, after crossing a combined area completely, she slowed down and stopped. After this series, each participant repeated the same procedure in the opposite direction by using other starting points (e.g., if the first series was S-down1, the second series was S-down2). The participants were instructed to walk at the center of the sidewalk.

Measurements. The velocity and tracks of the stroller, the pressure between the participant's palm and the stroller handle (PPH), and electromyograms (EMGs) of the participant's right upper limbs were measured throughout the stroller locomotion. When the participants moved forward to keep the right side of their body on the valley or mountain sides of the sidewalk, the measured upper limb was located at the downhill or uphill sides, respectively. Because the participants pushed the stroller forward in two directions, we could gather data of the PPH and EMGs in the upper limbs on both the downhill and the uphill sides. Under the combined slope condition, we analyzed the variables in two areas: going down (Down area) and going up (Up area) on the connecting area.

The behaviors of stroller locomotion were recorded by a video camera with a wide-angle lens (NV-GS100K, Panasonic, Japan), which was installed $2.8 \mathrm{~m}$ above the surface of the sidewalk. LED lights were attached on both casters of the stroller. Using $2 \mathrm{D}$ motion analysis software (Move-tr-32/2D, Library Inc., Japan), the center of the two markers was tracked at a sampling rate of $60 \mathrm{~Hz}$ to analyze the traveling velocity and track of the stroller. The starting point was set to the origin of the coordinates, and the lateral movement was calculated. The movements to the downhill and the uphill sides were expressed as plus and minus directions, respectively.

The PPH was measured by an air pack sensor (AMI Techno Co., 3037-2B, Japan). The size of the sensor was length $40 \mathrm{~mm}$, width $8 \mathrm{~mm}$, and thickness $1 \mathrm{~mm}$. The sensor was put on the right handle. The EMGs in five muscles (deltoid at the anterior, middle, and posterior areas; biceps brachii; triceps brachii; flexor carpi radialis; extensor carpi ulnaris) in the right upper limb were measured by a multi-purpose telemeter (Synaact MT-11, NEC-Sanei, Japan), whose data was acquired by recording software in a personal computer (Chart V4.2.3, ADInstrument, Australia) at a sampling rate of $1 \mathrm{kHz}$ via an A/D converter (Power Lab/8S, ADInstrument, Australia). We obtained the means of the absolute EMG waves (mEMG) and then expressed the mEMGs relative to that of the flat condition.

Statistical Analysis. All data were presented as mean \pm standard deviation. For the velocity of stroller locomotion and the $\mathrm{PPH}$, we performed a repeated-measure one-way analysis of variance (ANOVA) with the slope gradient as a factor. When a significant effect was found, Tukey's HSD method was performed. For the mEMG, we conducted the Friedman test with the slope gradient as a factor. When a significant effect was found, a Wilcoxon test was conducted. The accepted significance was $\mathrm{p}<0.05$.

\section{Results}

Traveling velocity and tracks of stroller locomotion. Under the cross slope condition and the combined slope condition at the Up area, the traveling velocity of the stroller decreased with the slope gradient. The velocity at a gradient of $15 \%$ was significantly lower than that of $0 \%$ in both conditions (Fig. 5). Under the cross slope condition at the Down area, the velocity at gradients of 5\% and 10\% increased significantly in comparison with that at the gradient of $0 \%$, although no significant increase was found in that of $15 \%$.

Figures 6, 7, and 8 show a participant's individual tracks under the cross slope condition and the combined slope conditions at the Up and Down areas, respectively. In all locomotion at gradients of $10 \%$ and $15 \%$, the strollers for most of the participants were driven off course and toward the downhill direction. Under the combined slope condition at the Down area, the stroller of some participants showed a drift of approximately 20 to $30 \mathrm{~cm}$ to the downhill direction. In the combined slope at the Up area, especially at the $15 \%$ gradient, most participants experienced rapid changes of course at the slope. 

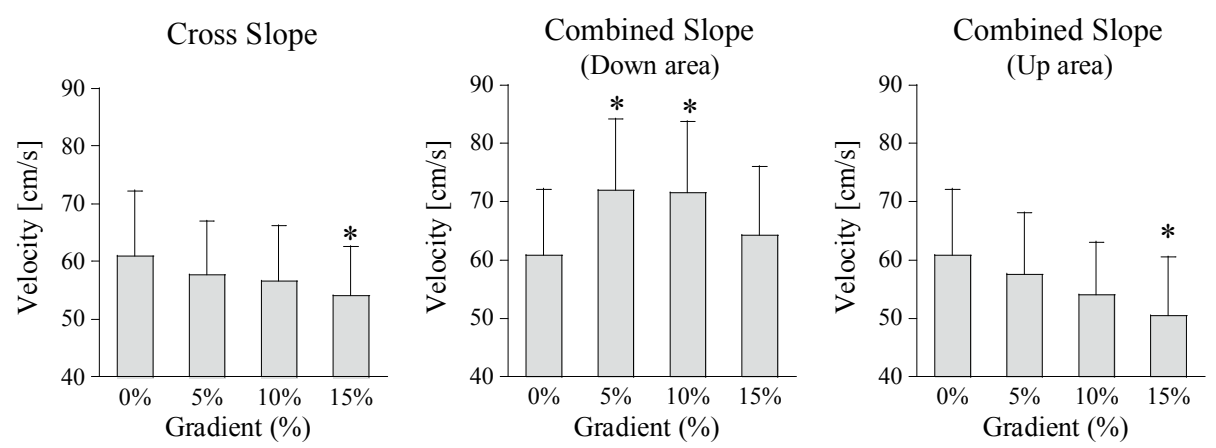

Fig. 5. Relationships between slope gradient and mean traveling velocity under the cross slope condition and the combined slope conditions at the Down and Up areas. * indicates a significant difference against the $0 \%$ gradient.
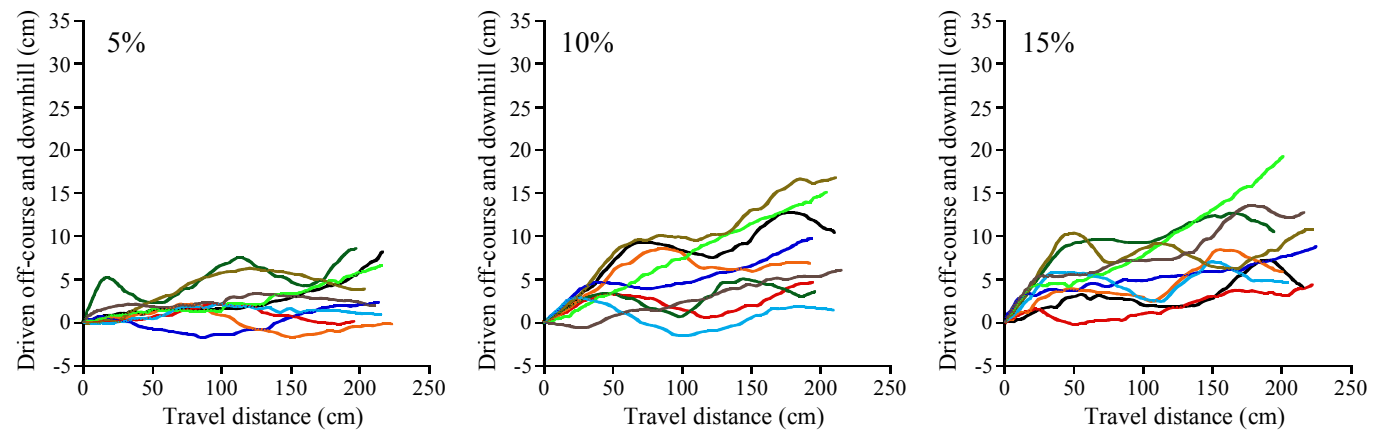

Fig. 6. Tracks of stroller locomotion for individual participants under the cross slope condition. The starting position is $\mathrm{x}(0)$.
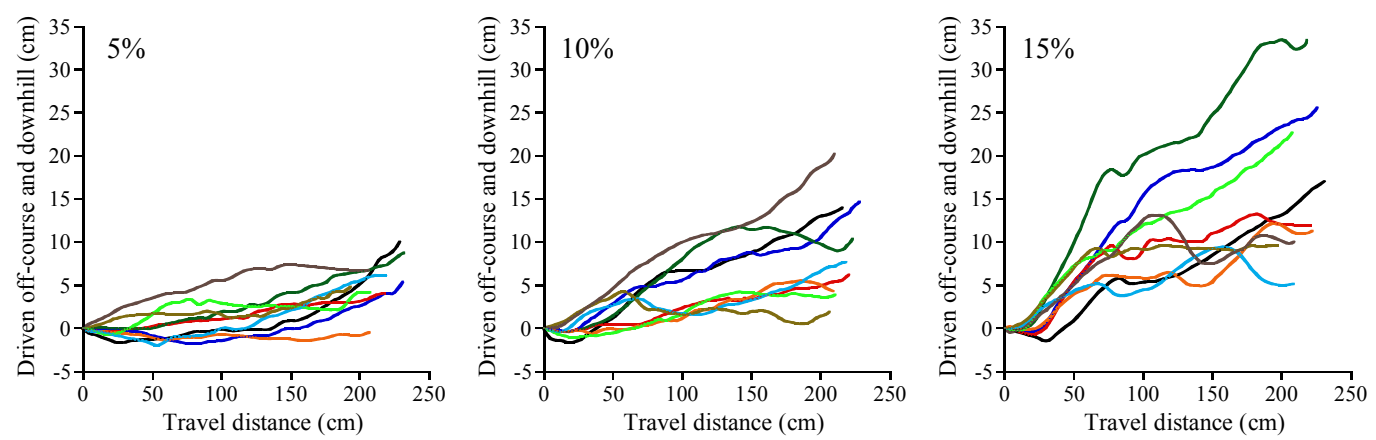

Fig. 7. Tracks of stroller locomotion for individual participants under the combined slope condition at the Down area. The starting position is $\mathrm{x}(0)$.
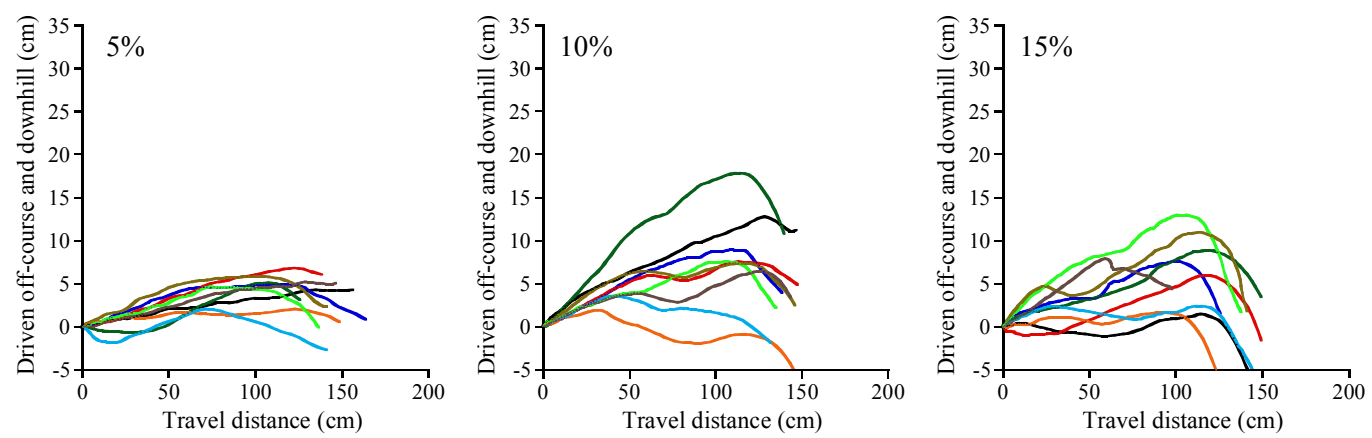

Fig. 8. Tracks of stroller locomotion for individual participants under the combined slope condition at the Up area. The starting position is $\mathrm{x}(0)$.

PPH and mEMG. In all conditions, the PPH increased with the slope gradient (Fig. 9). On both sides of the handle, significant increases were found at the higher gradient under all conditions. Under the combined condition at the Up area, the $\mathrm{PPH}$ at the $15 \%$ gradient became two times higher in 
comparison with that of the $0 \%$ gradient on both sides of the handle. The mEMG in most measured sites tended to increase with the slope gradient in all conditions, although only significant increases were found in the extensor carpi ulnaris because of great individual differences of the mEMGs (Fig. 10). In the combined slope at the Up and Down areas, significant increases at most gradients were found in the mEMGs of the extensor carpi ulnaris at the uphill side of the handle.
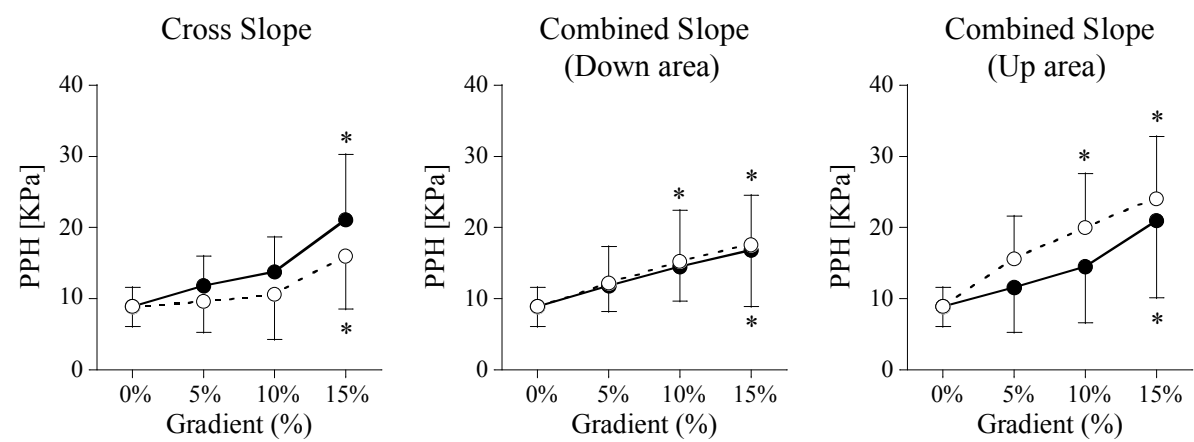

Fig. 9. Relationships between slope gradient and pressure between the palm and handle (PPH) under the cross slope condition and the combined slope conditions at the Down and Up areas. Open circles are the downhill side of the handle, and closed circles are the uphill side of the handle. ${ }^{*}$ indicates a significant difference against the $0 \%$ gradient.
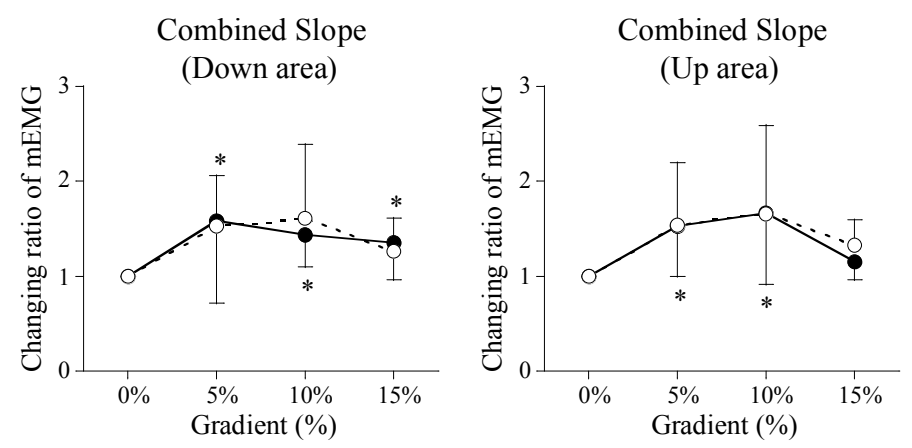

Fig. 10. Relationships between slope gradient and mEMG of the extensor carpi ulnaris under the cross slope condition and the combined slope conditions at the Down and Up areas. Closed circles are the uphill side of the handle. * indicates a significant difference against the $0 \%$ gradient.

\section{Discussion}

Under the cross slope condition, the drifts of stroller locomotion to the downhill direction were increased when the slope gradient was $10 \%$ and $15 \%$. Some participants could not restrain the drift of their strollers, especially at the gradient of $15 \%$, irrespective of approaching the edge of the sidewalk at the downhill side. These phenomena indicate that the participants could not control the stroller to stay on the intended course. One of the main causes of the drift is considered to be the continuous acceleration of stroller movement to the downhill direction by the tilt sideways. To prevent this drift, the participants naturally applied some strategies. As shown in Fig. 5, they decreased the traveling velocity according to the slope gradient. If they did not decrease the velocity, the drift would increase further. In addition, they gripped the handle more strongly with both hands, as shown in the results of the PPH (Fig. 9). The sideways tilt tended to turn the direction of casters toward the downhill direction; consequently, the handle also turned to the downhill direction. Although the participants applied these strategies, their effectiveness was not enough to prevent the drift completely. Therefore, a longer cross slope at a steeper gradient should be prohibited, because it promotes a continuing drift to the downhill direction.

In the combined slope at the Down area, the participants indicated a greater drift of locomotion and a greater physical effort in comparison with those of the cross slope condition. When the stroller entered the Down area, the caster at the downhill side was subjected to the combined slope first. This 
forced the stroller to rapidly tilt sideways to the downhill direction, then to accelerate the drift. In Fig. 7, sharp drifts were observed immediately for most participants after entering the area, especially at the $15 \%$ slope gradient, in spite of the participants' efforts to moderate the increase in velocity (Fig. 5). The significant increases in the mEMG in the extensor carpi ulnaris at the uphill side were considered to be muscle activities contributing to the reduction of the rapid drift. The combined area included two kinds of slopes, namely, the longitudinal and the cross slopes, whose gradients were determined by not only the gradient of the cross slope area but also the length of the combined area (Fig. 2). In the present study, it was fixed to $900 \mathrm{~mm}$, based on the results of our field survey. In this survey, a shorter length of the combined area was often observed in streets with a lot of vehicle parking. In these cases, the rapid drift at the beginning of the combined slope increased and therefore increased the danger of stroller locomotion.

In contrast, in the combined slope at the Up area, the locomotion velocity decreased with the slope gradient. Steeper gradients of the cross slope area also strengthened the gradient of the longitudinal slope in the combined slope area. Muraki et al. [5] studied wheelchair locomotion on longitudinal slopes at various gradients and indicated that the participants' selected velocities decrease with the gradient. In the mock sidewalk in the present study, the gradient of the cross slope at $15 \%$ produces a longitudinal gradient at $25 \%$ (height of lowest point (1500 x 0.15)/length of combined slope area (900)). Moreover, significant increases were found in the PPH and mEMG of the extensor carpi ulnaris. These results suggested that the physical strain was caused by the efforts to climb up the longitudinal gradient as well as to control the direction of the handles. Furthermore, rapid changes of the course direction toward the uphill side were observed when passing the end of the combined slope. At the combined slope at the Up area, stroller participants had to strongly control the stroller to the front and uphill direction in order to climb up the slope while lessening the movement to the downhill direction. When the stroller passed the end of the combined slope, the route was replaced by a flat surface, which reduced the necessary propulsion for stroller locomotion. Consequently, excess propulsion of the stroller is considered to lead to a rapid course change.

In the experiment of the present study, only one participant at a time was on the sidewalk. In a real situation, pedestrians with their strollers have to pass other pedestrians also on the sidewalk without contact between them. Furthermore, in a real situation, many circumstances arise for stroller users to operate the handle by using only one hand, for example, in the case that one hand grasps the hand of another child or some type of baggage. These situations on the cross and combined slopes present more difficulties in controlling the course, which could cause an accident [6]. The danger as indicated by the present study would increase in real conditions.

In conclusion, the findings of the present study suggested that installations of cross and combined slopes with higher slope gradients expose stroller users and the baby in the stroller to more danger. It is very difficult for them to keep their intended course, even if they decrease their locomotion velocity and make their greater physical effort to keep it in the right direction.

Acknowledgement. This study was supported by the Mitsui Sumitomo Insurance Welfare Foundation.

\section{Reference}

[1] W.J. Hurd, M.M. Morrow, K.R. Kaufman, K.N. An, Biomechanic evaluation of upper-extremity symmetry during manual wheelchair propulsion over varied terrain, Arch. Phys. Med. Rehabil. 89 (2008) 1996-2002.

[2] W.M. Richter, R. Rodriguez, K.R. Woods, P.W. Axelson, Consequences of a cross slope on wheelchair handrim biomechanics, Arch. Phys. Med. Rehabil. 88 (2007) 76-80.

[3] R.A. Cooper, E.E. Teodorski, M.L. Sporner, D.M. Collins, Manual wheelchair propulsion over cross-sloped surfaces: a literature review, Assist. Technol. 23 (2011) 42-51.

[4] K. Kockelman, L. Heard, Y.J. Kweon, T.W. Rioux, Sidewalk cross-slope design: Analysis of accessibility for persons with disabilities. Transportation Research Record, Transport Res. Rec. 1818 (2002) 108-118. 
[5] S. Muraki, S. Saito, A. Mihoshi, Physiological approach to a desirable gradient of longitudinal slope for all users. The Proceeding of the 2nd International Conference for Universal Design in Kyoto, (2006) CD-ROM.

[6] H. Ogami, S. Saito, M. Okura, S. Muraki, Investigation of physical strain and danger in sidewalk in stroller users. - Focusing on cross and compound slope and level difference-. Proceeding of Joint Conference of Engineering Applications on Wellbeing and Life Support 2005, (2005) 108. (in Japanese)

[7] Ordinance of the Japanese Ministry of Land, Infrastructure, Transport and Tourism. Act on promotion of smooth transportation, etc. of elderly persons, disabled persons, etc. (Law number: Act No. 91 of 2006), (2006).

[8] American with disabilities Act (ADA). ADA accessibility guidelines for buildings and facilities (ADAAG), (2002). 